\title{
Polytrauma Defined by the New Berlin Definition: A Validation Test Based on Propensity-Score Matching Approach
}

\author{
Cheng-Shyuan Rau ${ }^{1,+}{ }^{+}$, Shao-Chun $\mathrm{Wu}^{2,+}{ }^{+}$, Pao-Jen Kuo ${ }^{3}$, Yi-Chun Chen ${ }^{3}$, Peng-Chen Chien ${ }^{3}$, \\ Hsiao-Yun Hsieh ${ }^{3}$ and Ching-Hua Hsieh ${ }^{3, *}$ \\ 1 Department of Neurosurgery, Kaohsiung Chang Gung Memorial Hospital and Chang Gung University \\ College of Medicine, No.123, Ta-Pei Road, Niao-Song District, Kaohsiung City 833, Taiwan; \\ ersh2127@cloud.cgmh.org.tw \\ 2 Department of Anesthesiology, Kaohsiung Chang Gung Memorial Hospital and Chang Gung University \\ College of Medicine, No.123, Ta-Pei Road, Niao-Song District, Kaohsiung City 833, Taiwan; \\ shaochunwu@gmail.com \\ 3 Department of Plastic Surgery, Kaohsiung Chang Gung Memorial Hospital and Chang Gung University \\ College of Medicine, No.123, Ta-Pei Road, Niao-Song District, Kaohsiung City 833, Taiwan; \\ bow110470@gmail.com (P.-J.K.); libe320@yahoo.com.tw (Y.-C.C.); VENU_CHIEN@hotmail.com (P.-C.C.); \\ sylvia19870714@hotmail.com (H.-Y.H.) \\ * Correspondence: m93chinghua@gmail.com; Tel.: +886-7-7327476 \\ + Equal contribution in authorship.
}

Received: 9 August 2017; Accepted: 9 September 2017; Published: 11 September 2017

\begin{abstract}
Background: Polytrauma patients are expected to have a higher risk of mortality than that obtained by the summation of expected mortality owing to their individual injuries. This study was designed to investigate the outcome of patients with polytrauma, which was defined using the new Berlin definition, as cases with an Abbreviated Injury Scale (AIS) $\geq 3$ for two or more different body regions and one or more additional variables from five physiologic parameters (hypotension [systolic blood pressure $\leq 90 \mathrm{mmHg}$ ], unconsciousness [Glasgow Coma Scale score $\leq 8$ ], acidosis [base excess $\leq-6.0$ ], coagulopathy [partial thromboplastin time $\geq 40 \mathrm{~s}$ or international normalized ratio $\geq 1.4$ ], and age [ $\geq 70$ years]). Methods: We retrieved detailed data on 369 polytrauma patients and 1260 non-polytrauma patients with an overall Injury Severity Score (ISS) $\geq 18$ who were hospitalized between 1 January 2009 and 31 December 2015 for the treatment of all traumatic injuries, from the Trauma Registry System at a level I trauma center. Patients with burn injury or incomplete registered data were excluded. Categorical data were compared with two-sided Fisher exact or Pearson chi-square tests. The unpaired Student $t$-test and the Mann-Whitney $U$-test was used to analyze normally distributed continuous data and non-normally distributed data, respectively. Propensity-score matched cohort in a 1:1 ratio was allocated using the NCSS software with logistic regression to evaluate the effect of polytrauma on patient outcomes. Results: The polytrauma patients had a significantly higher ISS than non-polytrauma patients (median (interquartile range Q1-Q3), 29 (22-36) vs. 24 (20-25), respectively; $p<0.001$ ). Polytrauma patients had a 1.9-fold higher odds of mortality than non-polytrauma patients (95\% CI 1.38-2.49; $p<0.001)$. Compared to non-polytrauma patients, polytrauma patients had a substantially longer hospital length of stay (LOS). In addition, a higher proportion of polytrauma patients were admitted to the intensive care unit (ICU), spent longer LOS in the ICU, and had significantly higher total medical expenses. Among 201 selected propensity score-matched pairs of polytrauma and non-polytrauma patients who showed no significant difference in sex, age, co-morbidity, AIS $\geq 3$, and Injury Severity Score (ISS), the polytrauma patients had a significantly higher mortality rate (OR 17.5, 95\% CI 4.21-72.76; $p<0.001)$, and a higher proportion of patients admitted to the ICU $(84.1 \%$ vs. $74.1 \%$, respectively; $p=0.013)$ with longer stays in the ICU (10.3 days vs. 7.5 days, respectively; $p=0.003$ ). The total medical expenses for polytrauma patients were
\end{abstract}


$35.1 \%$ higher than those of non-polytrauma patients. However, there was no significant difference in the LOS between polytrauma and non-polytrauma patients (21.1 days vs. 19.8 days, respectively; $p=0.399$ ). Conclusions: The findings of this propensity-score matching study suggest that the new Berlin definition of polytrauma is feasible and applicable for trauma patients.

Keywords: polytrauma; new Berlin definition; Abbreviated Injury Scale (AIS); Glasgow Coma Scale (GCS); Injury Severity Score (ISS)

\section{Background}

The term "polytrauma" has been frequently defined in terms of a high Injury Severity Score (ISS) and has been generally used interchangeably with terms such as "severely injured" or "multiple trauma" [1]. The internationally accepted threshold of an ISS $\geq 16$ is based on the description as being predictive of a mortality risk above $10 \%$ [2]. However, a number of definitions of polytrauma with various ISS values (ISS $>15$ [3], ISS $>16$ [4], ISS $>18$ [5], ISS $\geq 18$ [6], or ISS $>25$ [7]) have been reported in the literature. In addition, a high ISS may be attributed to a severe single-system injury (monotrauma) rather than "polytrauma," which refers to trauma patients whose injuries involve multiple body regions and in whom the combination of injuries would cause a life-threatening condition $[8,9]$. If this concept is considered, polytrauma patients are expected to have a higher mortality rate than that obtained by the summation of expected mortality owing to their individual injuries [10], and to have more expensive therapeutic requirements [11], to require intensive resources for resuscitation, and to stay longer in the intensive care unit (ICU) [10].

Without a clear definition of polytrauma, any attempt to compare the loads, interventions, and outcomes of polytrauma patients among various trauma centers is challenging [10]. Some authors have suggested that at least two anatomical regions have to be injured for a patient to be identified as having polytrauma [9,12-14]. The 'polytrauma' definition of Butcher and colleagues using the Abbreviated Injury Scale (AIS) $\geq 3$ for at least two different body regions seemed more reasonable and feasible for identifying polytrauma patients $[1,10,14]$. With a higher mortality, more frequent ICU admissions, and longer hospital and ICU stays, this definition acts as a better predictor of morbidity and mortality than the definition using an ISS $>15$ or ISS $>17[1,10,14]$.

However, the definition of polytrauma based on the number of injured body regions does not reflect the physiological course after injury, which can be very dynamic in nature and may profoundly influence outcomes. Paffrath et al. even had reported that the mortality rate of polytrauma patients with an AIS $\geq 3$ for at least two different body regions was even lower (18.7\%) than that of patients with an ISS $\geq 16(20.4 \%)$ [11]. Our unpublished study also revealed that under the definition of polytrauma by AIS $\geq 3$ for at least two body regions, there was no significant difference in short-term mortality between polytrauma and non-polytrauma patients-i.e., polytrauma, as defined by AIS $\geq 3$ for at least two body regions, was not a distinguishing factor for recognizing a significant difference in short-term mortality among trauma patients. To improve the specificity of the polytrauma definition, some additional qualifying criteria have been proposed, such as the requirement of laparotomy [15], existence of severe shock [16], involvement of at least one vital organ necessitating admission into the ICU [17], and systemic inflammatory response syndrome on at least one day during the first $72 \mathrm{~h} \mathrm{[10].} \mathrm{However,} \mathrm{these} \mathrm{additional} \mathrm{criteria} \mathrm{seemed} \mathrm{to} \mathrm{be} \mathrm{limited} \mathrm{and} \mathrm{unverified.} \mathrm{Moreover,} \mathrm{the} \mathrm{levels}$ of variation and indication may differ among trauma centers.

The addition of a relevant physiologic condition or pathophysiologic change in combination of AIS/ISS is reasonable to increase its predictive power for mortality. Age, systolic blood pressure (SBP) and Glasgow Coma Scale (GCS) have been reported to have good predictive power for mortality [18]. An international consensus meeting in 2012 first tried to define polytrauma by combining the concept of injuries in different body regions and parameters of physiological response $[19,20]$. With the 
addition of at least one of five standardized physiological responses (hypotension [SBP $\leq 90 \mathrm{mmHg}$, unconsciousness [GCS score $\leq 8$ ], acidosis [base excess $\leq-6.0$ ], coagulopathy [partial thromboplastin time $\geq 40 \mathrm{~s}$ or international normalized ratio $\geq 1.4]$, and age $[\geq 70$ years]) in this new "Berlin definition" to the definition of ISS $\geq 16$ and AIS $\geq 3$ for at least two body regions, an improved definition of polytrauma was determined [13]. Notably, in the study that defines polytrauma as AIS $\geq 3$ points for two or more different body regions, mortality was $11.4 \%$ and $11.0 \%$ in polytrauma and non-polytrauma patients, respectively. A mortality rate of $18.7 \%$ was found when polytrauma was defined using ISS $\geq 16$ [13] and the mortality rates were increased to as high as $35-38 \%$ as soon as one other physiologic parameter was added [13].

Before applying the definition of the polytrauma in the clinical setting, we designed this study to investigate the outcome of polytrauma patients, with polytrauma being defined by the new Berlin definition, who admitted and treated for all trauma injuries at a level I trauma center. The primary hypothesis of this study was that polytrauma patients have a worse outcome than patients with similar injury severity but without polytrauma. In this study-for the assessment of the effect of polytrauma on the outcomes-we compared the selected propensity score-matched groups of patients to minimize confounding effects due to non-randomized assignment of patients into the polytrauma or non-polytrauma groups.

\section{Methods}

\section{Study Design}

This study was approved by the Institutional Review Board (IRB) of Kaohsiung Chang Gung Memorial Hospital (reference number 201600544B0), a level I regional trauma center providing care to trauma patients primarily from southern Taiwan [21,22]. Informed consent was waived according to IRB regulations. This retrospective study reviewed data of all hospitalized patients registered in the Trauma Registry System from 1 January 2009 to 31 December 2015. All patients with an overall ISS $\geq 18$ who were admitted for treatment of traumatic injuries were included and allocated into a polytrauma group or non-polytrauma group. The choice of 18 as a threshold of ISS for non-polytrauma patients depends on the consideration that the polytrauma patients at least would have an ISS of $18\left(3^{2}+3^{2}=18\right)$. The new Berlin definition of polytrauma was used and defined as follows [13]: a patient with AIS $\geq 3$ for two or more different body regions with additional one or more variables from the five physiologic parameters, including SBP $\leq 90 \mathrm{~mm} \mathrm{Hg}$, GCS score $\leq 8$, base excess $\leq 6.0$, international normalized ratio $\geq 1.4$ or partial thromboplastin time $\geq 40 \mathrm{~s}$, and age $\geq 70$ years.

The patients who had an overall ISS $\geq 18$ but did not fit into the above criteria of polytrauma were defined as non-polytrauma patients. Patients with burn injury or incomplete registered data were excluded. Detailed patient information retrieved from the Trauma Registry System included the following: age; gender; trauma mechanism; initial GCS score in the emergency department (ED); vital signs assessed by the physician upon arrival at the ED and procedures performed by the physician at the ED (cardiopulmonary resuscitation, intubation, insertion of chest tube, and blood transfusion); co-morbidities, such as diabetes mellitus (DM), hypertension (HTN), coronary artery disease (CAD), congestive heart failure (CHF), cerebral vascular accident (CVA), and end-stage renal disease (ESRD); AIS severity score for each body region; ISS; rates of associated injuries; hospital length of stay (LOS); the rates of admission into the ICU as well as the LOS in ICU; in-hospital mortality; and total medical expenses, which included the cost of examination (physical examination, radiography examination, hematology testing, pathological examination, electrocardiography examination, endoscopy, echocardiogram, electromyography, cardiac catheterization, and electroencephalography monitoring), cost of operation (operation fee and operation supply fee), cost of pharmaceuticals (medical service, narcotics, and medicine), and other costs (fees for administrative tasks, registration, wards, nursing, blood/plasma tests, anesthesia, hemodialysis, rehabilitation, special material costs, and personal expenses), expressed as cost per victim in US dollars. The ISS is expressed as the median 
and interquartile range (IQR). Odds ratios (ORs) of the associated conditions and injuries of the patients were presented with $95 \%$ confidence intervals (CIs). The primary outcome of the study was in-hospital mortality, and the secondary outcomes were hospital LOS, ICU admission rate, ICU LOS, and the total medical expenses. The analysis was performed using IBM SPSS Statistics for Windows, version 20.0 (IBM Corp., Armonk, NY, USA). Two-sided Fisher exact or Pearson chi-square tests were used to compare categorical data. The unpaired Student $t$-test and Mann-Whitney $U$-test were used to analyze normally distributed continuous and non-normally distributed data, respectively, which was reported as mean \pm standard deviation. To minimize confounding effects due to non-randomized assignment of patients into the polytrauma or non-polytrauma group, propensity score-matched groups of patients were selected for the assessment of the effect of polytrauma on the outcomes. A logistic regression model was used to calculate the propensity scores with the following covariates: gender; age, comorbidities, injury regions with AIS $\geq 3$, and ISS. A 1:1 matched study group was created by the Greedy method with a 0.2 caliper width using NCSS 10 software (NCSS Statistical software, Kaysville, UT, USA). After adjustment of these confounding factors, binary logistic regression was used for evaluating the effect of polytrauma on the primary and secondary outcomes. $p$-values $<0.05$ were considered statistically significant.

\section{Results}

\subsection{Injury Characteristics and Severity of Polytrauma Patients}

After the exclusion of patients with an ISS less than $18(n=18,017)$, with a burn injury $(n=896)$, or incomplete registered data $(n=129)$ from 20,106 hospitalized patients, there were 369 and 1260 patients in the polytrauma and non-polytrauma group, respectively (Figure 1). No significant differences in sex, age, pre-existing commodities, and injury mechanism were found between the polytrauma and non-polytrauma patients (Table 1). GCS scores were significantly lower for polytrauma patients than for non-polytrauma patients $(9.2 \pm 4.6$ vs. $11.8 \pm 4.2$, respectively; $p<0.001$ ). Significantly more polytrauma patients had a GCS $\leq 8$ than non-polytrauma patients. Analysis of injured body regions under the criteria of AIS $\geq 3$, revealed that polytrauma patients had sustained significantly higher rates of face, thoracic, abdominal, and extremity injuries than non-polytrauma patients, while no difference in head and neck injury was found between polytrauma and non-polytrauma patients. The polytrauma patients had a significantly higher ISS than non-polytrauma patients (median (IQR: Q1-Q3), 29 (22-36) vs. 24 (20-25), respectively; $p<0.001$ ). In addition, more polytrauma patients had an ISS $\geq 25$ and fewer patients had an ISS of 18-24 as compared to non-polytrauma patients.

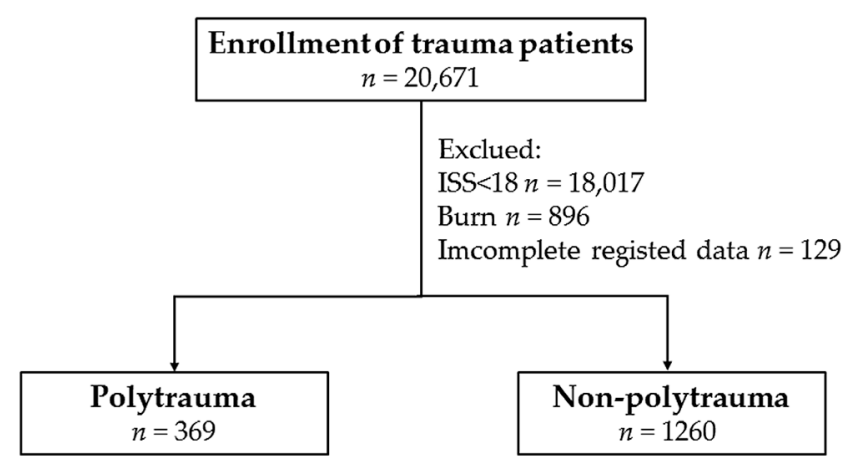

Figure 1. Flow chart of the studied polytrauma and non-polytrauma population. 
Table 1. Demographics and injury characteristics of polytrauma and non-polytrauma patients.

\begin{tabular}{|c|c|c|c|c|}
\hline Variables & Polytrauma $n=369$ & Non-Polytrauma $n=1260$ & Odds Ratio (95\% CI) & $p$ \\
\hline Sex & & & & 0.610 \\
\hline Male & $255(69.1)$ & $853(67.7)$ & $1.1(0.83-1.37)$ & \\
\hline Female & $114(30.9)$ & $407(32.3)$ & $0.9(0.73-1.20)$ & \\
\hline Age & $49.9 \pm 22.9$ & $47.3 \pm 19.9$ & - & 0.052 \\
\hline \multicolumn{5}{|l|}{ Comorbidity } \\
\hline DM & $37(10.0)$ & $169(13.4)$ & $0.7(0.49-1.05)$ & 0.085 \\
\hline HTN & $79(21.4)$ & $275(21.8)$ & $1.0(0.74-1.29)$ & 0.865 \\
\hline CAD & $15(4.1)$ & $36(2.9)$ & $1.4(0.78-2.66)$ & 0.241 \\
\hline $\mathrm{CHF}$ & $1(0.3)$ & $3(0.2)$ & $1.1(0.12-10.98)$ & 1.000 \\
\hline CVA & $5(1.4)$ & $30(2.4)$ & $0.6(0.22-1.46)$ & 0.232 \\
\hline ESRD & $0(0.0)$ & $1(0.1)$ & - & 1.000 \\
\hline \multicolumn{5}{|l|}{ Mechanism, n (\%) } \\
\hline MV Driver & $19(5.1)$ & $39(3.1)$ & $1.7(0.97-2.98)$ & 0.061 \\
\hline MV Passenger & $11(3.0)$ & $19(1.5)$ & $2.0(0.95-4.26)$ & 0.064 \\
\hline Motorcycle Driver & $222(60.2)$ & $792(62.9)$ & $0.9(0.70-1.13)$ & 0.348 \\
\hline Motorcycle Pillion & $18(4.9)$ & $38(3.0)$ & $1.6(0.93-2.93)$ & 0.084 \\
\hline Bicycle & $10(2.7)$ & $53(4.2)$ & $0.6(0.32-1.26)$ & 0.190 \\
\hline Pedestrian & $20(5.4)$ & $48(3.8)$ & $1.4(0.85-2.47)$ & 0.174 \\
\hline Fall & $59(16.0)$ & $230(18.3)$ & $0.9(0.62-1.17)$ & 0.317 \\
\hline Penetrating injury & $1(0.3)$ & $2(0.2)$ & $1.7(0.16-18.90)$ & 0.537 \\
\hline Struck by/against & $9(2.4)$ & $39(3.1)$ & $0.8(0.38-1.63)$ & 0.512 \\
\hline GCS & $9.2 \pm 4.6$ & $11.8 \pm 4.2$ & - & $<0.001$ \\
\hline$\leq 8$ & $200(54.2)$ & $301(23.9)$ & $3.8(2.96-4.80)$ & $<0.001$ \\
\hline $9-12$ & $32(8.7)$ & $181(14.4)$ & $0.6(0.38-0.84)$ & 0.004 \\
\hline$\geq 13$ & $137(37.1)$ & $778(61.7)$ & $0.4(0.29-0.47)$ & $<0.001$ \\
\hline \multicolumn{5}{|l|}{ AIS $\geq 3, \mathrm{n}(\%)$} \\
\hline Head/Neck & $281(76.2)$ & $940(74.6)$ & $1.1(0.83-1.43)$ & 0.546 \\
\hline Face & $19(5.1)$ & $12(1.0)$ & $5.6(2.71-11.74)$ & $<0.001$ \\
\hline Thorax & $237(64.2)$ & $378(30.0)$ & $4.2(3.28-5.35)$ & $<0.001$ \\
\hline Abdomen & $82(22.2)$ & $136(10.8)$ & $2.4(1.74-3.20)$ & $<0.001$ \\
\hline Extremity & $183(49.6)$ & $191(15.2)$ & $5.5(4.26-7.11)$ & $<0.001$ \\
\hline ISS, median (IQR) & $29(22-36)$ & $24(20-25)$ & - & $<0.001$ \\
\hline $18-24$ & $100(27.1)$ & $727(57.7)$ & $0.3(0.21-0.35)$ & $<0.001$ \\
\hline$\geq 25$ & $269(72.9)$ & $533(42.3)$ & $3.7(2.84-4.74)$ & $<0.001$ \\
\hline Mortality, n (\%) & $80(21.7)$ & $164(13.0)$ & $1.9(1.38-2.49)$ & $<0.001$ \\
\hline Hospital LOS (days) & $20.0 \pm 16.8$ & $16.8 \pm 15.6$ & - & 0.001 \\
\hline \multicolumn{5}{|l|}{ ICU } \\
\hline Patients, n (\%) & $298(80.8)$ & $951(75.5)$ & $1.4(1.02-1.82)$ & 0.035 \\
\hline $18-24$ & $66(22.1)$ & $479(50.4)$ & $0.3(0.21-0.38)$ & $<0.001$ \\
\hline$\geq 25$ & $232(77.9)$ & $472(49.6)$ & $3.6(2.64-4.82)$ & $<0.001$ \\
\hline LOS in ICU (days) & $10.1 \pm 9.5$ & $8.3 \pm 9.5$ & - & 0.005 \\
\hline Medical expenses & $8888 \pm 8141$ & $6270 \pm 6915$ & - & $<0.001$ \\
\hline Cost of examination & $591 \pm 536$ & $411 \pm 493$ & - & $<0.001$ \\
\hline Cost of operation & $1103 \pm 1198$ & $815 \pm 1084$ & - & $<0.001$ \\
\hline Cost of pharmaceuticals & $791 \pm 1291$ & $500 \pm 1028$ & - & $<0.001$ \\
\hline
\end{tabular}

\subsection{Outcomes of Polytrauma Patients}

Polytrauma patients had 1.9-fold higher odds of mortality than non-polytrauma patients (95\% CI 1.38-2.49; $p<0.001)$. Compared with non-polytrauma patients, polytrauma patients had significantly longer hospital LOS (20.0 days vs. 16.8 days, respectively; $p<0.001)$ and longer LOS in the ICU (10.1 days vs. 8.3 days, respectively; $p=0.005$ ). Moreover, compared to non-polytrauma patents, a higher proportion of polytrauma patients were admitted to the ICU $(80.8 \%$ vs. $75.5 \%$, respectively; $p=0.035$ ). In addition, the polytrauma patients spent a significantly higher amount on medical expenses ( $41.8 \%$ higher), examinations ( $43.8 \%$ higher), operations ( $35.3 \%$ higher), and pharmaceuticals (58.2\% higher) than non-polytrauma patients. 


\subsection{Associated Management and Injuries of Polytrauma Patients}

Polytrauma patients had significantly higher odds for worse hemodynamic measures and the requirement of procedures at the ED than non-polytrauma patients (Table 2). These measures included an SBP of $<90 \mathrm{mmHg}$, heart rate of $>100$ beats $/ \mathrm{min}$, and respiratory rate of $<10$ or $>29$ times $/ \mathrm{min}$. The required procedures included cardiopulmonary resuscitation, intubation, insertion of chest tube, and blood transfusion. Polytrauma patients had significantly higher ORs for sustaining subarachnoid hemorrhage (OR 1.4, 95\% CI 1.08-1.73; $p=0.010$ ) but lower ORs for subdural hematomas (OR 0.7, $95 \%$ CI $0.57-0.91 ; p=0.006$ ) than non-polytrauma patients (Table 3). In addition, polytrauma patients had significantly higher ORs for sustaining trauma in the thoracic, abdominal, and extremity regions than non-polytrauma patients.

Table 2. Physiological response and procedures performed upon arrival at the emergency department for polytrauma and non-polytrauma patients.

\begin{tabular}{ccccc}
\hline Variables & Polytrauma $\boldsymbol{n = 3 6 9}$ & Non-Polytrauma $\boldsymbol{n = 1 2 6 0}$ & Odds Ratio $\mathbf{( 9 5 \% ~ C I ) ~}$ & $p$ \\
\hline Physiology at ED, $\mathrm{n}(\%)$ & & & & \\
SBP $<90 \mathrm{mmHg}$ & $80(21.7)$ & $50(4.0)$ & $6.7(4.60-9.76)$ & $<0.001$ \\
$\mathrm{HR}>100$ beats $/ \mathrm{min}$ & $154(41.7)$ & $358(28.4)$ & $1.8(1.42-2.30)$ & $<0.001$ \\
$\mathrm{RR}<10$ or $>$ 29 times $/$ min & $36(9.8)$ & $29(2.3)$ & $4.6(2.77-7.60)$ & $<0.001$ \\
\hline Procedures at ED, $\mathrm{n}(\%)$ & & & & \\
Cardiopulmonary resuscitation & $15(4.1)$ & $11(0.9)$ & $4.8(2.19-10.57)$ & $<0.001$ \\
Intubation & $90(24.4)$ & $187(14.8)$ & $1.9(1.39-2.46)$ & $<0.001$ \\
Chest tube insertion & $59(16.0)$ & $84(6.7)$ & $2.7(1.87-3.80)$ & $<0.001$ \\
Blood transfusion & $145(39.3)$ & $132(10.5)$ & $5.5(4.20-7.29)$ & $<0.001$ \\
\hline
\end{tabular}

$\mathrm{ED}=$ emergency department; $\mathrm{HR}$ = heart rate; $\mathrm{RR}$ = respiratory rate; $\mathrm{SBP}$ = systolic blood pressure.

Table 3. Significant associated injuries in polytrauma and non-polytrauma patients.

\begin{tabular}{|c|c|c|c|c|}
\hline Variables & Polytrauma $n=369$ & Non-Polytrauma $n=1260$ & Odds Ratio (95\% CI) & $p$ \\
\hline \multicolumn{5}{|l|}{ Head trauma, n (\%) } \\
\hline Neurologic deficit & $14(3.8)$ & $33(2.6)$ & $1.5(0.78-2.77)$ & 0.236 \\
\hline Cranial fracture & $100(27.1)$ & $349(27.7)$ & $1.0(0.75-1.26)$ & 0.821 \\
\hline Epidural hematoma (EDH) & $74(20.1)$ & $310(24.6)$ & $0.8(0.58-1.02)$ & 0.070 \\
\hline Subdural hematoma (SDH) & $155(42.0)$ & $631(50.1)$ & $0.7(0.57-0.91)$ & 0.006 \\
\hline Subarachnoid hemorrhage (SAH) & $157(42.5)$ & $443(35.2)$ & $1.4(1.08-1.73)$ & 0.010 \\
\hline Intracerebral hematoma (ICH) & $46(12.5)$ & $129(10.2)$ & $1.2(0.87-1.79)$ & 0.224 \\
\hline Cerebral contusion & $71(19.2)$ & $261(20.7)$ & $0.9(0.68-1.22)$ & 0.537 \\
\hline \multicolumn{5}{|l|}{ Maxillofacial trauma, n (\%) } \\
\hline Orbital fracture & $10(2.7)$ & $55(4.4)$ & $0.6(0.31-1.21)$ & 0.153 \\
\hline Maxillary fracture & $53(14.4)$ & $195(15.5)$ & $0.9(0.66-1.27)$ & 0.601 \\
\hline Mandibular fracture & $15(4.1)$ & $48(3.8)$ & $1.1(0.59-1.93)$ & 0.823 \\
\hline \multicolumn{5}{|l|}{ Thoracic trauma, n (\%) } \\
\hline Rib fracture & $141(38.2)$ & $337(26.7)$ & $1.7(1.33-2.16)$ & $<0.001$ \\
\hline Hemothorax & $59(16.0)$ & $89(7.1)$ & $2.5(1.76-3.56)$ & $<0.001$ \\
\hline Pneumothorax & $50(13.6)$ & $107(8.5)$ & $1.7(1.18-2.42)$ & 0.004 \\
\hline Hemopneumothorax & $53(14.4)$ & $92(7.3)$ & $2.1(1.49-3.05)$ & $<0.001$ \\
\hline Lung contusion & $48(13.0)$ & $72(5.7)$ & $2.5(1.68-3.63)$ & $<0.001$ \\
\hline \multicolumn{5}{|l|}{ Abdominal trauma, n (\%) } \\
\hline Hepatic injury & $57(15.4)$ & $103(8.2)$ & $2.1(1.45-2.90)$ & $<0.001$ \\
\hline Splenic injury & $46(12.5)$ & $47(3.7)$ & $3.7(2.40-5.62)$ & $<0.001$ \\
\hline Retroperitoneal injury & $9(2.4)$ & $8(0.6)$ & $3.9(1.50-10.21)$ & 0.006 \\
\hline Renal injury & $13(3.5)$ & $25(2.0)$ & $1.8(0.91-3.56)$ & 0.085 \\
\hline \multicolumn{5}{|l|}{ Extremity trauma, n (\%) } \\
\hline Clavicle fracture & $54(14.6)$ & $224(17.8)$ & $0.8(0.57-1.10)$ & 0.158 \\
\hline Humeral fracture & $26(7.0)$ & $54(4.3)$ & $1.7(1.04-2.74)$ & 0.031 \\
\hline Radial fracture & $38(10.3)$ & $78(6.2)$ & $1.7(1.16-2.61)$ & 0.007 \\
\hline Ulnar fracture & $22(6.0)$ & $54(4.3)$ & $1.4(0.85-2.36)$ & 0.179 \\
\hline Pelvic fracture & $43(11.7)$ & $82(6.5)$ & $1.9(1.28-2.80)$ & 0.001 \\
\hline Femoral fracture & $99(26.8)$ & $86(6.8)$ & $5.0(3.64-6.88)$ & $<0.001$ \\
\hline Patella fracture & $15(4.1)$ & $18(1.4)$ & $2.9(1.46-5.86)$ & 0.002 \\
\hline Tibia fracture & $58(15.7)$ & $59(4.7)$ & $3.8(2.59-5.57)$ & $<0.001$ \\
\hline Fibular fracture & $43(11.7)$ & $44(3.5)$ & $3.6(2.35-5.65)$ & $<0.001$ \\
\hline
\end{tabular}




\subsection{Adjusted Outcomes of Polytrauma Patients in Propensity Score-Matched Patient Population}

A propensity score-matched patient population was selected to reduce the impact of demographic differences, pre-existing co-morbidities, and injury severity of the patient population on the outcome assessment between polytrauma and non-polytrauma patients (Table 4). In these 201 selected, well-balanced pairs of patients, there were no significant differences in sex, age, co-morbidity, number of patients with AIS $\geq 3$, and ISS. The logistic regression analysis of these pairs of patients showed that polytrauma patients had significantly higher mortality (OR 17.5, 95\% CI 4.21-72.76; $p<0.001$ ) than non-polytrauma patients. Compared with non-polytrauma patients, the polytrauma patients had a higher proportion of patients admitted to the ICU ( $84.1 \%$ vs. $74.1 \%$, respectively; $p=0.013)$ and a longer stay in ICU (10.3 days vs. 7.5 days, respectively; $p=0.003)$, but there was no significant difference in the hospital LOS (21.1 days vs. 19.8 days, respectively; $p=0.399$ ). In addition, polytrauma patients still had a significantly higher total medical expense ( $35.1 \%$ higher), cost of examination (33.1\% higher), cost of operation (40.6\% higher), and cost of pharmaceuticals (53.9\% higher) than the non-polytrauma patients.

Table 4. Covariates and the assessment of outcomes in polytrauma and non-polytrauma patients adjusted in 1:1 Greedy propensity-score matching.

\begin{tabular}{|c|c|c|c|c|}
\hline Variables & Polytrauma $n=201$ & Non-Polytrauma $n=201$ & Odds Ratio (95\%) & $p$ \\
\hline Sex & & & & 1.000 \\
\hline Male & $149(74.1)$ & $149(74.1)$ & $1.0(0.64-1.56)$ & \\
\hline Female & $52(25.9)$ & $52(25.9)$ & $1.0(0.64-1.56)$ & \\
\hline Age & $43.2 \pm 20.0$ & $43.4 \pm 16.4$ & - & 0.919 \\
\hline \multicolumn{5}{|l|}{ Comorbidity } \\
\hline $\mathrm{DM}$ & $12(6.0)$ & $12(6.0)$ & $1.0(0.44-2.28)$ & 1.000 \\
\hline HTN & $27(13.4)$ & $27(13.4)$ & $1.0(0.56-1.77)$ & 1.000 \\
\hline CAD & $0(0.0)$ & $0(0.0)$ & - & - \\
\hline $\mathrm{CHF}$ & $0(0.0)$ & $0(0.0)$ & - & - \\
\hline CVA & $0(0.0)$ & $0(0.0)$ & - & - \\
\hline ESRD & $0(0.0)$ & $0(0.0)$ & - & - \\
\hline \multicolumn{5}{|l|}{ AIS $\geq 3, n(\%)$} \\
\hline Head/Neck & 144 (71.6) & 144 (71.6) & $1.0(0.65-1.54)$ & 1.000 \\
\hline Face & $5(2.5)$ & $5(2.5)$ & $1.0(0.29-3.51)$ & 1.000 \\
\hline Thorax & $133(66.2)$ & $133(66.2)$ & $1.0(0.66-1.51)$ & 1.000 \\
\hline Abdomen & $45(22.4)$ & $45(22.4)$ & $1.0(0.63-1.60)$ & 1.000 \\
\hline Extremity & $99(49.3)$ & $99(49.3)$ & $1.0(0.68-1.48)$ & 1.000 \\
\hline ISS, median (IQR) & $27(22-34)$ & $26(22-29)$ & - & 0.271 \\
\hline Mortality, n (\%) & $35(17.4)$ & $2(1.0)$ & $17.5(4.21-72.76)$ & $<0.001$ \\
\hline Hospital LOS (days) & $21.1 \pm 16.2$ & $19.8 \pm 14.4$ & - & 0.399 \\
\hline \multicolumn{5}{|l|}{ ICU } \\
\hline Patients, n (\%) & $169(84.1)$ & $149(74.1)$ & $2.0(1.15-3.30)$ & 0.013 \\
\hline LOS in ICU (days) & $10.3 \pm 9.3$ & $7.5 \pm 7.4$ & - & 0.003 \\
\hline Medical expenses & $9634 \pm 8850$ & $7129 \pm 6800$ & - & 0.002 \\
\hline Cost of examination & $627 \pm 584$ & $471 \pm 516$ & - & 0.005 \\
\hline Cost of operation & $1225 \pm 1238$ & $871 \pm 1039$ & - & 0.002 \\
\hline Cost of pharmaceutical & $851 \pm 1431$ & $553 \pm 1085$ & - & 0.019 \\
\hline
\end{tabular}

\section{Discussion}

This study compared clinical outcomes in a broad group of hospitalized polytrauma and non-polytrauma patients to investigate the feasibility and applicability of the new Berlin definition of polytrauma. We found that polytrauma patients presented with significantly higher morbidity and mortality than non-polytrauma patients. More importantly, even after consideration of the differences in demographic characteristics, co-morbidities, and injury severity of the trauma patient population, 
the selected propensity score-matched polytrauma patients still had a significantly higher proportion of patients admitted to the ICU with a longer stay, a higher total medical cost, and higher mortality than non-polytrauma patients.

Polytrauma is generally used to describe trauma patients whose injuries involve multiple body regions, compromise the patient's physiology and potentially cause dysfunction of uninjured organs [13]. The expected higher risk of mortality of polytrauma patients is based on the assumption that the underlying pathophysiological response of the injured person would aggravate the clinical outcome. The injured person's pathophysiological response to the injury load, however, makes a differentiation between "polytrauma" and "multitrauma" [10]. For example, many reports had indicated that head and brain injuries and thoracic traumata are major risk factors in trauma patients and that co-occurrence of these factors leads to an exponential increase in mortality [23-25]. The reduced pulmonary reserves in lung contusion may rapidly lead to hypoventilation and hypoxia that cause secondary injury to the brain or pose more burden to other organ systems. Early intubation was also a significant risk factor for cerebral impairment in patients with multiple trauma [26]. The injury to the gastrointestinal system may interfere with the nutrition balance and increase bacterial translocation $[27,28]$ - moreover, increasing levels of endotoxemia following polytrauma have been reported [29]. Likewise, hypovolemic shock and massive blood transfusion are often associated with coagulopathy and imbalance in acid-base homeostasis [30]. Notably, in this study polytrauma patients had statistically significant higher ORs for sustaining thoracic trauma and abdominal trauma as well as for requiring procedures including cardiopulmonary resuscitation, intubation, chest tube insertion, and blood transfusion at the ED. These combined scenarios may explain partly, but not enough, our observation of a higher mortality in the selected propensity score-matched polytrauma patients than that in non-polytrauma patients.

A combination of injury severity, relevant pathophysiologic change, or physiologic changes seemed be useful for mortality prediction [13]. The dysregulation of the immune system after trauma presents one of the greatest threats to life [31,32]. However, a formally defined pathophysiological response to trauma remains a challenge. In addition, even in a prospective study, the practicability of including systemic inflammatory response syndrome into the definition of polytrauma as a surrogate for physiological derangement was questioned [33]. In contrast, the selected five physiological parameters (hypotension [SBP $\leq 90 \mathrm{mmHg}$ ], unconsciousness [GCS score $\leq 8$ ], acidosis [base excess $\leq-6.0$ ], coagulopathy [partial thromboplastin time $\geq 40 \mathrm{~s}$ or international normalized ratio $\geq 1.4$ ], and age $[\geq 70$ years]) of the new "Berlin definition" for polytrauma are deemed feasible worldwide and easily approachable with less ambiguity [13]. This study based on a propensity-score matching approach may provide more evidence to support the use of such a definition of polytrauma in clinical settings to manage with polytrauma patients.

In this study, the non-polytrauma group is composed of two different types of patients: first, patients with only one body region affected (AIS $\geq 4$ ), with or without physiological problems; and second, patients with two or more body regions affected, but no physiological problem. The matching used in this study is prone to select the latter group of patients for comparison, thus may have a selection bias. This study has some other limitations that should be acknowledged. First, the retrospective design of the study would carry an inherent selection bias. Second, the patients declared dead at the accident scene or on hospital arrival were not included in the Trauma Registry Database, which may have led to a selection bias. Third, as the registered data lacked uniform indication of hospitalization and admission into ICU, as well as the type of surgery performed on patients, we could only rely on the assumption that management of patients with or without polytrauma was uniform. Further, long-term mortality was not evaluated in this study. Finally, the physiological problems also work in isolated trauma [11]. With an increasing number of physiological factors there was an almost linear increase in mortality up to an $86 \%$ rate in patients with all five physiological factors present [11]. In this study, the addition of physiological factor(s) to the polytrauma but not to non-polytrauma patients, making the present non-polytrauma group is a 
mixture of two groups with separate injury pattern, i.e. one with, and one without, a physiological problem. Therefore, a selection bias may be existed in the comparison.

\section{Conclusions}

As we noted a significantly higher incidence of morbidity and mortality in polytrauma patients than in non-polytrauma patients, our study findings based on propensity-score matching validate the new definition of polytrauma based on the new Berlin definition, as a case with AIS $\geq 3$ for two or more different body regions with additional one or more variables from the five physiologic parameters.

Author Contributions: Cheng-Shyuan Rau wrote the manuscript, Shao-Chun Wu was involved in the review of literature, Pao-Jen Kuo was responsible for the integrity of registered data, Yi-Chun Chen performed the statistical analyses, Hsiao-Yun Hsieh proofread the manuscript, and Ching-Hua Hsieh designed the study and contributed to the analysis and interpretation of data. All authors read and approved the final manuscript.

Conflicts of Interest: The authors declare that they have no competing interests.

\section{References}

1. Butcher, N.E.; D'Este, C.; Balogh, Z.J. The quest for a universal definition of polytrauma: A trauma registry-based validation study. J. Trauma Acute Care Surg. 2014, 77, 620-623. [CrossRef] [PubMed]

2. Boyd, C.R.; Tolson, M.A.; Copes, W.S. Evaluating trauma care: The TRISS method. Trauma Score and the Injury Severity Score. J. Trauma 1987, 27, 370-378. [CrossRef] [PubMed]

3. Sikand, M.; Williams, K.; White, C.; Moran, C.G. The financial cost of treating polytrauma: Implications for tertiary referral centres in the United Kingdom. Injury 2005, 36, 733-737. [CrossRef] [PubMed]

4. Biewener, A.; Aschenbrenner, U.; Rammelt, S.; Grass, R.; Zwipp, H. Impact of helicopter transport and hospital level on mortality of polytrauma patients. J. Trauma 2004, 56, 94-98. [CrossRef] [PubMed]

5. Hildebrand, F.; Giannoudis, P.; Kretteck, C.; Pape, H.C. Damage control: Extremities. Injury 2004, 35, 678-689. [CrossRef] [PubMed]

6. Pape, H.C.; Remmers, D.; Rice, J.; Ebisch, M.; Krettek, C.; Tscherne, H. Appraisal of early evaluation of blunt chest trauma: Development of a standardized scoring system for initial clinical decision making. J. Trauma 2000, 49, 496-504. [CrossRef] [PubMed]

7. McLain, R.F. Functional outcomes after surgery for spinal fractures: Return to work and activity. Spine (Phila Pa 1976) 2004, 29, 470-477. [CrossRef]

8. Tscherne, H. [The treatment of the seriously injured at an emergency station]. Chirurg 1966, 37, 249-252. [PubMed]

9. Border, J.R.; LaDuca, J.; Seibel, R. Priorities in the management of the patient with polytrauma. Prog. Surg. 1975, 14, 84-120. [PubMed]

10. Butcher, N.; Balogh, Z.J. The definition of polytrauma: The need for international consensus. Injury 2009, 40 (Suppl. 4), S12-S22. [CrossRef] [PubMed]

11. Paffrath, T.; Lefering, R.; Flohe, S. How to define severely injured patients?-An Injury Severity Score (ISS) based approach alone is not sufficient. Injury 2014, 45 (Suppl. 3), S64-S69. [CrossRef] [PubMed]

12. Tscherne, H.; Regel, G.; Sturm, J.A.; Friedl, H.P. Degree of severity and priorities in multiple injuries. Chirurg 1987, 58, 631-640. [PubMed]

13. Pape, H.C.; Lefering, R.; Butcher, N.; Peitzman, A.; Leenen, L.; Marzi, I.; Lichte, P.; Josten, C.; Bouillon, B.; Schmucker, U.; et al. The definition of polytrauma revisited: An international consensus process and proposal of the new 'Berlin definition'. J. Trauma Acute Care Surg. 2014, 77, 780-786. [CrossRef] [PubMed]

14. Butcher, N.; Balogh, Z.J. AIS $>2$ in at least two body regions: A potential new anatomical definition of polytrauma. Injury 2012, 43, 196-199. [CrossRef] [PubMed]

15. Cerra, F.B.; Mazuski, J.; Teasley, K.; Nuwer, N.; Lysne, J.; Shronts, E.; Konstantinides, F. Nitrogen retention in critically ill patients is proportional to the branched chain amino acid load. Crit. Care Med. 1983, 11, 775-778. [CrossRef] [PubMed]

16. Deby-Dupont, G.; Haas, M.; Pincemail, J.; Braun, M.; Lamy, M.; Deby, C.; Franchimont, P. Immunoreactive trypsin in the adult respiratory distress syndrome. Intensiv. Care Med. 1984, 10, 7-12. [CrossRef] 
17. Blacker, D.J.; Wijdicks, E.F. Clinical characteristics and mechanisms of stroke after polytrauma. Mayo Clin. Proc. 2004, 79, 630-635. [CrossRef] [PubMed]

18. Kondo, Y.; Abe, T.; Kohshi, K.; Tokuda, Y.; Cook, E.F.; Kukita, I. Revised trauma scoring system to predict in-hospital mortality in the emergency department: Glasgow Coma Scale, Age, and Systolic Blood Pressure score. Crit. Care 2011, 15, R191. [CrossRef] [PubMed]

19. Pape, H.C. Classification of patients with multiple injuries-Is the polytrauma patient defined adequately in 2012? Injury 2012, 43, 127-128. [CrossRef] [PubMed]

20. Pape, H.C.; Lefering, R. Grading of injury severity-What should be the prerequisites to separate multiply injured patients from those in critical condition and polytrauma? Injury 2013, 44, 157-158. [CrossRef] [PubMed]

21. Hsieh, C.H.; Hsu, S.Y.; Hsieh, H.Y.; Chen, Y.C. Differences between the sexes in motorcycle-related injuries and fatalities at a Taiwanese level I trauma center. Biomed. J. 2017, 40, 113-120. [CrossRef] [PubMed]

22. Hsieh, C.H.; Liu, H.T.; Hsu, S.Y.; Hsieh, H.Y.; Chen, Y.C. Motorcycle-related hospitalizations of the elderly. Biomed. J. 2017, 40, 121-128. [CrossRef] [PubMed]

23. Patel, V.I.; Thadepalli, H.; Patel, P.V.; Mandal, A.K. Thoracoabdominal injuries in the elderly: 25 years of experience. J. Natl. Med. Assoc. 2004, 96, 1553-1557. [PubMed]

24. Nirula, R.; Gentilello, L.M. Futility of resuscitation criteria for the "young" old and the "old" old trauma patient: A national trauma data bank analysis. J. Trauma 2004, 57, 37-41. [CrossRef] [PubMed]

25. Shorr, R.M.; Rodriguez, A.; Indeck, M.C.; Crittenden, M.D.; Hartunian, S.; Cowley, R.A. Blunt chest trauma in the elderly. J. Trauma 1989, 29, 234-237. [CrossRef] [PubMed]

26. Zietlow, S.P.; Capizzi, P.J.; Bannon, M.P.; Farnell, M.B. Multisystem geriatric trauma. J. Trauma 1994, 37, 985-988. [CrossRef] [PubMed]

27. Deitch, E.A.; Rutan, R.; Waymack, J.P. Trauma, shock, and gut translocation. New Horiz. 1996, 4, $289-299$. [PubMed]

28. Lichtman, S.M. Bacterial [correction of baterial] translocation in humans. J. Pediatr. Gastroenterol. Nutr. 2001, 33, 1-10. [CrossRef] [PubMed]

29. Charbonney, E.; Tsang, J.Y.; Li, Y.; Klein, D.; Duque, P.; Romaschin, A.; Marshall, J.C. Endotoxemia Following Multiple Trauma: Risk Factors and Prognostic Implications. Crit. Care Med. 2016, 44, 335-341. [CrossRef] [PubMed]

30. Sihler, K.C.; Napolitano, L.M. Complications of massive transfusion. Chest 2010, 137, 209-220. [CrossRef] [PubMed]

31. Miller, A.C.; Rashid, R.M.; Elamin, E.M. The "T" in trauma: The helper T-cell response and the role of immunomodulation in trauma and burn patients. J. Trauma 2007, 63, 1407-1417. [CrossRef] [PubMed]

32. Ni Choileain, N.; Redmond, H.P. The immunological consequences of injury. Surgeon 2006, 4, $23-31$. [CrossRef]

33. Butcher, N.E.; Balogh, Z.J. The practicality of including the systemic inflammatory response syndrome in the definition of polytrauma: Experience of a level one trauma centre. Injury 2013, 44, 12-17. [CrossRef] [PubMed]

(C) 2017 by the authors. Licensee MDPI, Basel, Switzerland. This article is an open access article distributed under the terms and conditions of the Creative Commons Attribution (CC BY) license (http://creativecommons.org/licenses/by/4.0/). 